\title{
Saliência, relevância e determinância da carne bovina para os consumidores de Dracena (SP): uma nova abordagem metodológica
}

\author{
Beef salience, relevance and determinance for consumers in \\ Dracena - São Paulo State: a new methodological approach \\ Etiénne Groot ${ }^{1}$ (D) Ródney Lúcio Pinheiro Henrique² (i)

\begin{abstract}
'Faculdade de Ciências Agrárias e Tecnológicas, Programa de Pós-graduação em Ciência e Tecnologia Animal, Universidade Estadual Paulista (UNESP), Dracena (SP), Brasil. E-mail: etienne.groot@unesp.br

${ }^{2}$ Faculdade de Engenharia, Programa de Pós-graduação em Agronomia, Universidade Estadual Paulista (UNESP), Ilha Solteira (SP),
\end{abstract} \\ Brasil. E-mail: rodney.lucioh@gmail.com
}

\begin{abstract}
Como citar: Groot, E., Henrique, R. L. P. (2022). Saliência, relevância e determinância da carne bovina para os consumidores de Dracena (SP): uma nova abordagem metodológica. Revista de Economia e Sociologia Rural, 60(4), e246908. https://doi.org/10.1590/1806-9479.2021.246908
\end{abstract}

Resumo: $\bigcirc$ objetivo deste estudo foi avaliar a saliência, a relevância e a determinância da carne bovina para os consumidores de Dracena (SP), por meio de dois métodos de mensuração. A saliência foi determinada pelo método de elicitação livre (EL), a relevância e a determinância foram calculadas pelo modelo multinomia logit, com dados de um experimento de escolha discreta de melhor-pior (EEDMP) do caso 2. Para comparar as determinâncias, as preferências também foram estimadas empregando-se o modelo Probit, mediante declarações de intenções de compra de carne bovina. No momento da compra, o atributo mais saliente é a cor da carne, ao passo que o sabor e a textura são os atributos mais salientes durante o consumo. Resultados mostram ainda discrepâncias entre a determinância obtida pelo EEDMP e pelas intenções de compra. As preferências expressadas pelas intenções de compra se aproximam mais da literatura especializada, o que indica que a carne saborosa, macia e vermelha brilhante é a mais apreciada entre os consumidores. Ainda é prematuro fazer recomendações do uso concatenado da EL e do EEDMP para estimar as três dimensões das características de um produto, como a carne bovina.

Palavras-chave: importância dos atributos, certificação da carne bovina, percepção de qualidade da carne, intenção de compra.

Abstract: This study aimed to evaluate the beef salience, relevance, and determinance for consumers in Dracena - São Paulo State (Brazil), through two measurement methods. The salience was determined by Free Elicitation Method (FEM), relevance and determinance were calculated using the Multinomial Logit Model, with Case 2 Best-Worst Discrete Choice Experiment (EEDMP) data. To compare the determinance, preferences were also estimated by the Probit model, based on beef purchase intentions. At the time of purchase, the most salient attribute is the meat's color, while the flavor and texture are the most salient attributes in the consumption. Results also show discrepancies between the determinance obtained by EEDMP and purchase intentions. The expressed preferences of purchasing intentions are closer to the specialized literature, which states that tasty, tender and bright red beef is the most appreciated among consumers. It is still premature to make recommendations on concatenated use of FEM and EEDMP to estimate the three characteristics' dimensions of a product, such as beef.

Keywords: attributes' importance, beef certification, beef quality perception, purchase intention.

\section{INTRODUÇÃO}

O Brasil é um grande player na produção e no consumo de carne bovina. Em 2019, o país possuía o maior rebanho de bovinos do mundo, com 213,7 milhões de cabeças. Naquele ano, foram abatidos 43,3 milhões de cabeças, totalizando 10,49 milhões de TEC (toneladas equivalentes de carcaça). Da produção total, $76,3 \%$ foram destinadas ao mercado interno, 
que ainda importou 47 mil TEC para atender ao consumo. O consumo per capita de carne bovina no Brasil foi estimado em 39,12 kg. Esse valor supera o consumo per capita de países como os EUA (37,24 kg/hab.) e Austrália (30,32 kg/hab.) (Associação Brasileira das Indústrias Exportadoras de Carne, 2020).

Mesmo tendo um grande mercado de carne bovina, o Brasil apresenta realidades extremamente discrepantes em toda a sua cadeia produtiva, com importantes implicações na qualidade do produto. Por um lado, há uma indústria altamente tecnificada e profissionalizada e, no outro extremo, encontram-se produtores descapitalizados e abatedouros clandestinos (Tirado, 2009). Mesmo existindo um segmento mais competitivo, o mercado spot da carne bovina comercializa a mercadoria como commodity, ou seja, os produtos não possuem diferenciação e apresentam baixo valor agregado (Aguiar, 2018). Para evitar a concorrência puramente baseada em custos e preços, Grunert et al. (2004) destacam a necessidade de ofertar novos produtos diferenciados, para aumentar a satisfação e a fidelidade dos consumidores.

Para identificar as oportunidades e melhorar o posicionamento dos produtos no mercado, é preciso conhecer os fatores que levam os consumidores a comprar um ou outro produto. Com base no conhecimento dos atributos de qualidade mais relevantes para os consumidores, os stakeholders envolvidos com a criação e a comercialização de bovinos podem desenvolver novos produtos que ofereçam um valor superior ao mercado consumidor.

Identificar e mensurar a importância das características desses produtos são informações críticas na pesquisa em marketing, uma vez que os atributos impactam a atitude do consumidor (Espinoza \& Hirano, 2003). Neste sentido, diversas pesquisas de opinião foram realizadas para estimar o grau de importância dos atributos da carne bovina no comportamento de consumo. Por exemplo, Garcia et al. (2018) estudaram, por meio da escala de soma constante, o grau de importância dos atributos intrínsecos e extrínsecos da carne bovina para os brasileiros. No Rio Grande do Sul, Ries (2010) verificou quanto valor os consumidores de Caxias do Sul, Antônio Prado e Nova Petrópolis atribuíam aos atributos da carne bovina, por meio da escala de Likert. No Chile, Schnettler et al. (2010) estudaram a importância de 21 atributos de qualidade da carne bovina nas decisões de compra dos consumidores de Temuco.

Mesmo que os trabalhos anteriormente mencionados tenham contribuído ao estudo das preferências dos consumidores, os métodos de pesquisa que adotaram avaliam apenas uma dimensão da importância dos atributos, por isso podem carecer de validade contingente e nomológica. Conforme descrito na próxima seção, a maioria dos trabalhos que investigam a importância dos atributos para os consumidores sofre de ausência de validade, que pode ocorrer por vários motivos, um deles seria o aumento da complexidade das pesquisas para avaliar todas as dimensões da importância dos atributos.

Neste sentido, o presente trabalho realizou duas contribuições significativas: uma com um aspecto mais prático e outra mais acadêmica/metodológica. Pela relevância do mercado nacional de carne bovina, o objetivo prático foi estudar as três dimensões da importância das características de qualidade da carne bovina: saliência, relevância e determinância, no comportamento de compra e consumo dos consumidores de Dracena. Esse município localizase no oeste do estado de São Paulo e sua população foi estimada em 47.043 pessoas, em 2020. Sua principal atividade econômica é a agropecuária. Em 2018, o PIB per capita foi estimado em 25.779,84 reais (Instituto Brasileiro de Geografia e Estatística, 2020).

O propósito acadêmico foi propor a mensuração das três dimensões da importância dos atributos de qualidade por meio da Elicitação Livre (EL) e do Experimento de Escolha Discreta de Melhor-Pior (EEDMP) do caso 2. O aspecto inovador nessa pesquisa é a mensuração das três dimensões da importância das características dos produtos, por meio de dois métodos de 
mensuração em vez de três, como foi proposto por Van Ittersum et al. (2007) para as pesquisas de marketing.

\section{FUNDAMENTAÇÃO TEÓRICA}

\subsection{MENSURAÇÃO DA IMPORTÂNCIA DOS ATRIBUTOS}

Existem vários procedimentos para determinar a importância dos atributos de um bem, não obstante muitos métodos possuem baixa validade convergente e validade nomológica, o que gera problemas empíricos e práticos. Segundo Jaccard et al. (1986), a validade convergente existe quando diferentes métodos geram resultados positivamente relacionados, ao passo que a análise possui validade nomológica apenas quando permite realizar previsões precisas.

Com isso em mente, Van Ittersum et al. (2007) estimaram a validade convergente de 91 artigos científicos, publicados nas principais revistas acadêmicas, e concluíram que $67 \%$ dos trabalhos usavam métodos com falta de validade. A falta de validade nomológica também foi verificada nesses artigos. Para esses autores, uma das causas dos problemas observados estava na abordagem unidimensional das pesquisas.

Os primeiros autores a propor a multidimensionalidade da importância das características dos bens e serviços foram Myers e Alpert (1977). Eles sugeriram a existência de três dimensões: saliência, relevância e determinância. Saliência foi definida com o artigo de Krech e Crutchfield (1948), que considera que "nem todas as crenças se destacam igualmente no campo cognitivo de um indivíduo. O indivíduo pode estar mais ciente de algumas crenças que de outras". De forma análoga, a facilidade na qual os atributos vêm à mente das pessoas ou de ser reconhecidos quando as pessoas pensam sobre algo determina sua saliência. A característica elicitada primeiro é a mais saliente e a característica mencionada na sequência é a segunda mais importante, e assim por diante.

De acordo com Myers e Alpert (1977), um atributo é importante ou relevante quando há consequências ou significado ao realizar uma escolha entre marcas ou produtos. Van Ittersum et al. (2007) complementam que a relevância do atributo é determinada pelos valores e desejos de cada pessoa.

Diferentemente da relevância, as características determinantes estão mais intimamente ligadas às preferências ou às decisões de compra reais (Myers \& Alpert, 1977). A determinância do atributo é avaliada pela influência que seus diferentes níveis exercem no julgamento e nas decisões de compra. Quanto maiores as diferenças entre os níveis do atributo, maiores sua determinância e, consequentemente, sua importância.

Para melhorar a validade convergente e nomológica de uma análise, Van Ittersum et al. (2007) sugerem que cada uma das três dimensões de importância das características de um bem ou serviço seja mensurada por metodologias específicas. Para mensurar a saliência, o método indicado deve omitir qualquer informação prévia sobre o atributo ao respondente, pois as pessoas devem recuperá-lo da memória. Sempre que o método fornece informações sobre os níveis dos atributos ao respondente, o mais indicado é medir a determinância do atributo; não obstante, se o respondente é notificado a respeito dos atributos, mas não acerca dos seus respectivos níveis, o método indicado deve medir a relevância dos atributos. As metodologias indicadas por Van Ittersum et al. (2007) para avaliar cada dimensão de importância dos atributos dos bens e serviços estão expostas na Tabela 1. 
Tabela 1. Metodologias indicadas para avaliar cada dimensão da importância dos atributos

\section{Dimensão}

Saliência

Relevância

\section{Metodologia}

Método de EL (free-elicitation method)

Método de rating direto (direct-rating method)

Método de ranking direto (direct-ranking method)

Método de alocação de ponto (point-allocation method)

Processo hierárquico analítico (analytical hierarchy process)

Placa de exibição de informações (information displayed board)

Determinância

Método de atitude multiatributo (multi-attribute attitude model)

Método trade-off (trade-off mode)

Método do balanço ponderado (swing-weight method)

Método do conjunto (conjoint method)

Fonte: autores segundo Van Ittersum et al. (2007)

Para Van Ittersum et al. (2007), são necessários três métodos de mensuração para avaliar - de forma integral - as três dimensões da importância dos atributos. Um questionário muito complexo ou extenso demanda mais esforço cognitivo e tempo de resposta do entrevistado. Ao responder ao questionário, o cansaço ocasionado pela complexidade das perguntas repercute negativamente na qualidade das respostas dos entrevistados. A duração da atividade também pode comprometer a qualidade das respostas. Estudos mostram que após 20 minutos os participantes começam a dedicar menos tempo em oferecer respostas e optam com mais frequência por respostas como "não sei" ou "não aplicável" (Levordashka, 2006). Dessa forma, a simplificação dos questionários passa a ser uma regra na pesquisa de opinião. A priori, se não há perda de conteúdo, é preferível adotar dois métodos de pesquisa em vez de três.

Neste sentido, o presente estudo foi realizado para avaliar, de forma completa, as três dimensões da importância dos atributos da carne bovina utilizando apenas dois métodos complementares: Elicitação Livre (EL) e Experimento de Escolha Discreta de Melhor-Pior (EEDMP) do caso 2. O método de EL avaliou a saliência dos atributos, conforme sugerido por Van Ittersum et al. (2007). O EEDMP do caso 2 estimou, numa mesma escala, a relevância dos atributos mais salientes e a determinância de seus respectivos níveis.

\subsection{EXPERIMENTO DE ESCOLHA DISCRETA DE MELHOR-PIOR}

O EEDMP foi proposto por Finn e Louviere (1992). A princípio, a ideia do uso do EEDMP era superar algumas limitações de outros métodos de estudo de preferências e também aproveitar a habilidade das pessoas de identificar opções extremas. Nesse experimento, o respondente indica a melhor (a que proporciona mais utilidade) e a pior (a que proporciona menos utilidade) alternativas num conjunto de escolha com, pelo menos, três opções.

De acordo com Aizaki \& Fogarty (2019), existem três tipos de EEDMP: o experimento do caso 1 (ou caso do objeto), do caso 2 (caso do perfil) e do caso 3 (caso do multiperfil). A fundamentação teórica de cada tipo de experimento foi publicada em diferentes artigos, tendo a do caso 2 sido formalizada por Marley et al. (2008). O experimento consiste em apresentar conjuntos/situações de escolha, que são diferentes perfis de produtos ou serviços, resultantes de diferentes combinações de níveis de atributos (Quadro 1). Em cada situação de escolha, o respondente deve indicar o melhor e o pior níveis de atributo que o levariam a comprar o produto ou o serviço. Embora pareça uma tarefa inusual, Marley et al. (2008) asseguram que o experimento proporciona resultados confiáveis e interpretáveis. 
Quadro 1. Situação de escolha do experimento

\begin{tabular}{cc} 
Característica da carne & $(+)$ \\
& $\mathrm{R} \$ 15 / \mathrm{kg}$ \\
Marrom & \\
& Bom sabor garantido \\
& Maciez garantida \\
Compraria a carne? ( ) Sim ( ) Não & \\
\hline
\end{tabular}

Fonte: a própria pesquisa.

O EEDMP do caso 2 tem sido amplamente adotado em pesquisas de opinião, principalmente na área de saúde e cuidados com a saúde (Mühlbacher et al., 2016). A informação adicional proporcionada pelas escolhas das piores alternativas, além de aumentar a eficiência dos modelos de escolha, reduz a necessidade de observações para a análise. Assim, o experimento permite realizar estudos de preferências com pessoas portadoras de doenças raras ou com profissionais especializados (Lancsar et al., 2013), em que o tamanho da população e o da amostra são limitados.

O aspecto essencial do experimento é que, ao escolher uma alternativa, há a indicação de um atributo e de seu respectivo nível, simultaneamente, numa escala comum, o que possibilita estimar a importância (relevância) relativa dos atributos, bem como a utilidade marginal (determinância) de seus respectivos níveis, separadamente (Marley \& Flynn, 2015). Neste sentido, o EEDMP do caso 2 pode substituir dois métodos de mensuração: um para estimar a relevância dos atributos e outro para calcular a determinância dos níveis de atributos.

\section{METODOLOGIA}

Todo atributo saliente é relevante e determinante em seus níveis, porém a relação inversa nem sempre é verdadeira (Van Ittersum et al., 2007). Desta forma, a avaliação da saliência dos atributos foi realizada antes da análise da relevância dos atributos e da determinância de seus respectivos níveis.

A pesquisa envolveu a aplicação de dois questionários. O primeiro visou estudar a saliência dos atributos da carne bovina por meio do Método de EL e o segundo questionário avaliou a relevância dos atributos mais salientes e a determinância de seus respectivos níveis pelo EEDMP do caso 2.

\subsection{MÉTODO DE ELICITAÇÃO LIVRE}

O método de EL consiste em fazer uma entrevista individual, em que se solicita ao entrevistado que verbalize seus primeiros pensamentos (recordações) em resposta a estímulos (Grebitus, 2008). A fundamentação teórica desse método está baseada na Teoria da Ativação de Propagação (Spreading Activation Theory) (Collins \& Loftus, 1975), que considera que as pessoas constroem estruturas de conhecimento durante a vida e são capazes de relatar verbalmente parte ou a maior parte de seu conteúdo armazenado na memória (Benedict et al., 1997).

Por se tratar de perguntas abertas, a interpretação das respostas é subjetiva. No entanto, os resultados foram analisados e classificados em categorias, tal como sugere Van Kleef et al. (2005). Entre as recomendações, assume-se que o primeiro atributo mencionado pelo entrevistado é o mais saliente, o segundo atributo é o segundo mais saliente e assim por diante. 
A pesquisa considerou o Modelo de Qualidade Total de Alimentos. Esse modelo descreve o processo de compra em dois períodos: um antes e outro depois da aquisição. Antes da compra, os consumidores formam suas expectativas sobre a qualidade do produto. Nessa etapa, não é possível avaliar os atributos de experiência, como o sabor e a textura do alimento. Depois da compra, os consumidores experimentam a qualidade do produto, seja pela preparação, seja pelo consumo da carne bovina (Grunert, 2005).

O questionário adotado na pesquisa continha duas perguntas: uma solicitando aos respondentes que relacionassem os atributos da carne bovina que mais consideram no momento da compra e outra pedindo que listassem os atributos que mais consideram depois da compra, ou seja, no momento da preparação e do consumo da carne bovina no dia a dia. Em ambos os casos, os entrevistados responderam livremente às indagações.

\subsection{EXPERIMENTO DE ESCOLHA DISCRETA DE MELHOR-PIOR DO CASO 2}

\subsubsection{Desenho experimental}

O primeiro passo do desenho experimental é definir os atributos e seus respectivos níveis. A escolha dos atributos deu-se por meio dos resultados da análise anterior de saliência dos atributos da carne bovina, obtidos pelo método EL. No total, foram incorporados quatro atributos no desenho experimental (preço, cor, sabor e maciez da carne) e atribuídos três níveis para cada atributo (Tabela 2). Por ser a carne mais consumida em Dracena (SP), o produto hipotético do experimento de escolha foi a alcatra.

A determinação dos níveis de preços da alcatra foi baseada numa pesquisa de preço nos principais supermercados da cidade. O preço médio foi calculado em $R \$ 25 / \mathrm{kg}$. O preço oscilou em até $R \$ 10,00 / \mathrm{kg}$. Portanto, o menor preço foi estipulado em $R \$ 15 / \mathrm{kg}$ e o maior, em $R \$ 35 / \mathrm{kg}$.

Tabela 2. Atributos da carne bovina (alcatra) e seus respectivos níveis

\begin{tabular}{|c|c|c|c|}
\hline Atributos & \multicolumn{3}{|c|}{ Níveis de atributos } \\
\hline Preço & $\mathrm{R} \$ 15 / \mathrm{kg}$ & $\mathrm{R} \$ 25 / \mathrm{kg}$ & $\mathrm{R} \$ 35 / \mathrm{kg}$ \\
\hline Cor da carne & Vermelho-brilhante & Vermelho-púrpura & Marrom \\
\hline Sabor da carne & Bom sabor garantido & $\begin{array}{l}\text { Provavelmente tem bom } \\
\text { sabor }\end{array}$ & $\begin{array}{l}\text { Provavelmente não tem } \\
\text { bom sabor }\end{array}$ \\
\hline Maciez da carne & Maciez garantida & Provavelmente macia & Provavelmente dura \\
\hline
\end{tabular}

Fonte: dados da pesquisa.

Os níveis de cor da carne bovina foram determinados com base no trabalho de Carpenter et al. (2001), que avaliaram a influência das cores nas decisões de compra de carne bovina em Utah, EUA. Outros trabalhos fizeram uso desses mesmos níveis de cores, entre eles os artigos de Passetti et al. (2016) e Andrighetto et al. (2010). Para proporcionar uma referência sobre as cores da carne bovina aos entrevistados, antes de responderem ao experimento de escolha, os três níveis de cores foram apresentados por meio de uma carta de cores (Figura 1).

A cor da carne tem um papel de extrema importância na avaliação da qualidade do alimento. Os consumidores se baseiam nessa variável para formar suas expectativas sobre a qualidade organoléptica do produto. O sabor e a textura são atributos de experiência por serem avaliados apenas no consumo do alimento. Inferir sobre o sabor ou a textura, apenas considerando a cor da carne, pode levar a erros na compra e gerar insatisfação entre os consumidores. 

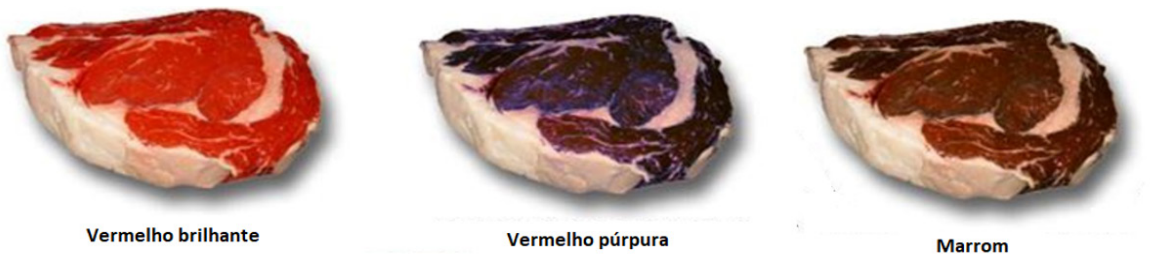

Figura 1. Níveis de cores da carne bovina. Fonte: elaboração dos autores com base em Carpenter et al. (2001).

Para minimizar o risco nas compras, o mercado tem investido em certificações (atributos de crença) para comprovar a qualidade do produto ao consumidor. No Brasil, existem iniciativas como a certificação Red Angus, que envolve toda a cadeia produtiva para oferecer ao mercado uma carne mais macia e saborosa (Flores, 2016). Nessa pesquisa, os níveis dos atributos sabor e maciez buscaram avaliar a preferência dos consumidores pela carne macia e saborosa, bem como a preferência pela garantia genérica (sem indicação de uma marca) dessas características de qualidade.

Uma vez determinados os atributos e seus níveis, foram distribuídos em diferentes produtos hipotéticos, resultantes de diferentes combinações dessas características (Quadro 1). As combinações foram baseadas num desenho ortogonal de efeito principal (orthogonal main effect plan), tal como sugere Flynn et al. (2007). Esse critério oferece as combinações necessárias para realizar estimativas tanto pela abordagem de pares como a marginal. $O$ desenho experimental obtido foi balanceado, em que cada nível de atributo aparece no desenho experimental com a mesma frequência. Todos os entrevistados responderam a nove situações de escolha.

Com o intuito de comparar os resultados das utilidades marginais obtidas pelo EEDMP do caso 2, após indicar o melhor nível de atributo (aquele que tornaria o produto mais atrativo para a compra) e o pior nível de atributo (aquele que tornaria o produto menos atrativo para a compra), solicitou-se ao respondente que indicasse sua intenção de compra daquele produto hipotético (pergunta na parte inferior do Quadro 1). 0 embasamento teórico para a realização das estimativas está descrito nas próximas seções.

\subsubsection{Modelo empírico EEDMP do caso 2}

Os EEDMPs baseiam-se na Teoria da Utilidade Aleatória (TUA), de Thurstone (Marley \& Louviere, 2005). De acordo com a TUA, o indivíduo ipercebe um benefício $\left(U_{b, i}\right)$ com a alternativa $b$ e esse benefício pode ser decomposto num componente sistemático $\left(v_{b, i}\right)$, que é observável pelo pesquisador, e num componente aleatório $\left(\varepsilon_{b, i}\right)$, que não é observável, conforme mostra a Equação 1.

$U_{b, i}=V_{b, i}+\varepsilon_{b, i}$

Assume-se o comportamento racional dos consumidores. Sendo assim, o consumidor $i$ escolherá a alternativa $b$ como a melhor alternativa entre Jalternativas disponíveis no conjunto de escolha, se e somente se, o benefício proporcionado pela alternativa $b$ for melhor que 0 benefício das demais alternativas (Equação 2). Da mesma maneira, o consumidor i escolherá a alternativa $w$ como a pior entre $J$ alternativas, se e somente se, o seu benefício for pior entre as alternativas disponíveis (Equação 3). 
$V_{b, i} \geq V_{j, i}$ para todo $j \neq b \in J$

$V_{w, i} \leq V_{j, i}$ para todo $j \neq w \in J$

A probabilidade de o indivíduo iescolher a alternativa $b$ como a melhor é dada pelas Equações 4 e 5 .

$P_{b, i}=\operatorname{Pr}\left(U_{b, i} \geq U_{j, i}, j=1,2, . ., J\right)$

$P_{b, i}=\operatorname{Pr}\left(V_{b, i}+\varepsilon_{b, i} \geq V_{j, i}+\varepsilon_{j, i}, j=1,2, . ., J\right)$

$P_{b, i}=\operatorname{Pr}\left(V_{b, i}-V_{j, i} \geq \varepsilon_{j, i}-\varepsilon_{b, i}, j=1,2, . ., J\right)$

A probabilidade de o indivíduo i escolher a alternativa wcomo a pior é dada pelas Equações 6 e 7.

$P_{b, i}=\operatorname{Pr}\left(U_{w, i} \leq U_{j, i}, j=1,2, . ., J\right)$

$P_{b, i}=\operatorname{Pr}\left(V_{w, i}+\varepsilon_{w, i} \leq V_{j, i}+\varepsilon_{j, i}, j=1,2, . ., J\right)$

$P_{b, i}=\operatorname{Pr}\left(V_{w, i}-V_{j, i} \leq \varepsilon_{j, i}-\varepsilon_{w, i}, j=1,2, . ., J\right)$

Admitindo-se que os termos aleatórios têm distribuição independente e idêntica, bem como valor extremo do tipo I (equivalente à distribuição Gumbel), o modelo logit condicionado pode ser calculado. Por meio da abordagem marginal das escolhas, a probabilidade de o indivíduo $i$ escolher, concomitantemente, a opção $b$ como a melhor e a opção $w$ como a pior pode ser estimada pela equação 8. Para efeitos práticos, nas estimativas dos modelos econométricos, as variáveis independentes categóricas e numéricas são multiplicadas por -1 (menos um) quando se trata das indicações das piores alternativas, tal como recomendado por Flynn et al. (2007).

$P_{b w, i}=\frac{\exp \left(V_{b, i}\right)}{\sum_{j=1}^{J} \exp \left(V_{j, 1}\right)} \times \frac{\exp \left(-V_{w, i}\right)}{\sum_{j=1}^{J} \exp \left(-V_{j, 1}\right)}$

O modelo estimado tem fundamentação na Teoria do Valor de Lancaster. A função é aditiva e linear, ou seja, considera que o benefício da alternativa $q$ é influenciado por todas as características do produto. Por se tratar de um EEDMP do caso 2, o benefício total que o indivíduo item com a alternativa qé a soma das utilidades marginais (determinâncias) dos níveis dos atributos (representadas pelos "a"s) e dos impactos (relevâncias) relativos dos atributos (descritos pelos " $\beta$ "s). Esses benefícios são capturados pela Equação 9. 


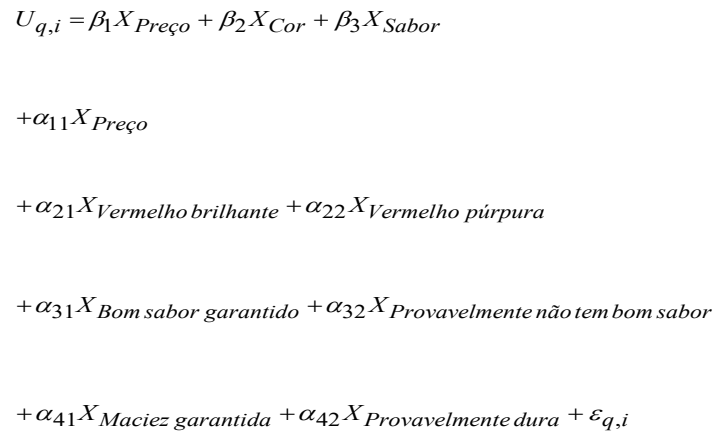

A codificação das variáveis seguiu as orientações de Flynn et al. (2007). Os níveis dos atributos qualitativos foram codificados com código de efeito e os atributos foram codificados com variáveis dummy. Os níveis de preço foram codificados numericamente.

\subsection{ANÁLISE PROBIT}

Nesta pesquisa, para avaliar as preferências dos consumidores de Dracena em comprar carne bovina, realizaram-se análises pelo modelo Probit. Esse modelo é um método estatístico com duas categorias de variáveis dependentes. Por um lado, a variável dependente binária assume valor 1 quando o consumidor indica que compraria a carne. Caso contrário, atribui-se valor 0 (zero). Por outro lado, o modelo considera que cada consumidor possui um nível crítico ou limiar chamado de $I_{i}^{*}$. Caso $I_{i}$ (que é o índice de utilidade não observável) seja maior que o nível crítico, o consumidor comprará a carne bovina. Do contrário, não (Gujarati, 2000). A análise permite mensurar, por exemplo, quanto as variações nos níveis dos atributos de alcatra aumentam ou diminuem a probabilidade de comprar o produto.

Admitindo-se a hipótese de normalidade, a probabilidade de $I_{i}^{*}$ ser menor que ou igual a $I_{i}$ pode ser representada pela função de distribuição acumulativa (FDA) normal padronizada de uma variável qualquer. Em outras palavras, a probabilidade de escolher qualquer alternativa em vez de não escolher pode ser expressa pela Equação 10, onde 。 representa a FDA.

$$
\begin{aligned}
& P_{i}=\operatorname{Pr}\left(Y_{i}=1 \mid X\right)=\operatorname{Pr}\left(I_{i}^{*} \leq I_{i}\right)=F\left(I_{i}\right)=\frac{1}{\sqrt{2 \pi}} \int_{-\infty}^{T_{i}} e^{-t^{2} / 2} d t \\
& P_{i}=\frac{1}{\sqrt{2 \pi}} \int_{-\infty}^{\beta_{1}+\beta_{2} X_{i}} e^{-t^{2} / 2} d t=\Phi\left(x_{i}^{\prime} \beta\right)
\end{aligned}
$$

A relação entre uma determinada variável e a mudança de probabilidade pode ser interpretada como o efeito marginal (Uzunoz \& Akcay, 2012). O efeito marginal ligado às variáveis independentes contínuas (como é o caso do preço da carne) com a probabilidade $\operatorname{Pr}\left(Y_{i}=1 \mid X\right)$, ceteris paribus, pode ser calculado pela Equação 11:

$$
\frac{\partial P_{i}}{\partial X_{i k}}=\Phi\left(x_{i}^{\prime} \beta\right) \beta_{k}
$$


Os efeitos marginais das variáveis dummy devem ser calculados de outra forma. As mudanças discretas nas probabilidades previstas constituem uma alternativa ao efeito marginal na avaliação da influência das variáveis categóricas, conforme mostra a Equação 12.

$\Delta=\Phi(\bar{x} \beta, d=1)-\Phi(\bar{x} \beta, d=0)$

Os efeitos marginais propiciam informações de como as variáveis explicativas impactam a probabilidade de consumo.

\subsection{AMOSTRAGEM E ANÁLISE DOS DADOS}

As duas amostragens foram realizadas em momentos diferentes. A primeira amostragem que visou mensurar a saliência dos atributos da carne bovina foi realizada de maio a julho de 2017. A segunda amostragem, cujo intuito era medir a relevância dos atributos mais salientes da carne bovina e determinar seus respectivos níveis, foi realizada no período de setembro a novembro de 2017.

Na primeira amostragem, foram feitas 60 entrevistas, das quais 51 foram consideradas válidas, e na segunda amostragem, houve a participação de 100 pessoas, tendo 97 entrevistas sido consideradas válidas. Para validar a entrevista, era necessário que o questionário não apresentasse erros de preenchimento.

As entrevistas foram feitas nas principais vias públicas da cidade de Dracena. Os consumidores foram convidados a participar da pesquisa de forma aleatória. Os erros amostrais foram calculados seguindo as instruções de Trespalacios et al. (2005). Deste modo, com um intervalo de confiança de $95 \%$, o erro amostral da primeira amostra foi estimado em $13,7 \%$ e o erro da segunda amostra foi calculado em 9,95\%. Por se tratar de um estudo exploratório, é possível aceitar mais riscos de imprecisão dos resultados, uma vez que o propósito do trabalho se restringe a gerar hipóteses e insights sobre o problema (Marotti et al., 2008).

As respostas das entrevistas foram inicialmente tabuladas em planilhas-cálculo. A saliência dos atributos foi calculada por meio de estatística descritiva, pelo programa IBM SPSS. A relevância dos atributos e a determinância dos níveis de atributos foram estimadas pelo Modelo Multinomial Logit. Indicações de intenção de compras serviram para estimar a determinância dos níveis de atributos pelo modelo Probit. Essas análises foram feitas com o software Nlogit.

\section{RESULTADOS E DISCUSSÕES}

\subsection{PERFIL SOCIODEMOGRÁFICO DOS CONSUMIDORES}

As características sociodemográficas dos consumidores que participaram das entrevistas dos dois questionários - e da população de Dracena estão descritas na Tabela 3. O primeiro questionário contou com a participação de 51 consumidores, enquanto o segundo, com 97 consumidores. A participação masculina foi predominante nos dois questionários, no entanto a participação masculina no primeiro $(64,7 \%)$ foi superior à do segundo questionário (57,7\%). É de conhecimento geral que os homens são coadjuvantes nas decisões de compra de alimentos em seus lares. De acordo com Lelis et al. (2012), 80\% dos responsáveis pelas atividades alimentares domésticas são mulheres, as grandes responsáveis pelas compras dos alimentos. No entanto, o tradicional churrasco é uma atividade mais relacionada ao homem, 
conferindo-Ihe envolvimento com os aspectos de qualidade da carne bovina. O engajamento das mulheres e dos homens com o produto é um aspecto desejável à pesquisa.

Em relação à idade, na média, os consumidores que responderam ao questionário 2 podem ser considerados mais jovens que a população de Dracena, pois, na amostra, há mais porcentagem de pessoas com até 30 anos de idade e menos proporção de pessoas com pelo menos 60 anos. No questionário 1, observa-se que a faixa etária predominante foi 30 a 59 anos.

Quanto aos estudos, as amostras representam, em maior proporção, preferências de pessoas com mais nível de estudos, ou seja, pessoas mais informadas. Praticamente, não há consumidores sem estudos nas amostras. Essa porcentagem para a população foi de $44,9 \%$ em 2010. Praticamente um terço dos consumidores entrevistados estava cursando ou havia concluído os estudos universitários, enquanto apenas $12,8 \%$ das pessoas em Dracena possuíam esse nível de estudos. Os anos de estudo, principalmente ter curso superior ou pós-graduação, impactam a renda familiar (Menezes Filho et al., 2016).

Os consumidores entrevistados tinham renda familiar muito superior à da população. Em 2010, apenas 4,7\% dos dracenenses pertenciam à classe social A ou B, ou seja, tinham renda familiar superior a cinco salários-mínimos ${ }^{1}$ (acima de $R \$ 4.865 /$ mês ou US\$1.437,78/mês). Entre entrevistados do questionário 1,39,2\% dos respondentes consideraram ser da classe social A ou $B$, enquanto $56,7 \%$ dos consumidores que responderam ao questionário 2 afirmaram ser da classe social A ou B. Portanto, espera-se que os entrevistados tenham mais poder aquisitivo que a média dos cidadãos de Dracena.

Tabela 3. Características sociodemográficas dos consumidores entrevistados

\begin{tabular}{|c|c|c|c|c|c|c|c|}
\hline \multirow{2}{*}{ Variável } & \multirow{2}{*}{ Categoria } & \multicolumn{2}{|c|}{ Questionário 1} & \multicolumn{2}{|c|}{ Questionário 2} & \multicolumn{2}{|c|}{ População ${ }^{3}$} \\
\hline & & Número & $\%$ & Número & $\%$ & Número & $\%$ \\
\hline \multirow[t]{2}{*}{ Gênero } & Masculino & 33 & 64,7 & 56 & 57,7 & 15.992 & 49,0 \\
\hline & Feminino & 18 & 35,3 & 41 & 42,3 & 16.625 & 51,0 \\
\hline \multirow[t]{3}{*}{ Faixa etária } & Menos de 30 anos & 9 & 17,6 & 25 & 25,8 & 7.037 & 21,6 \\
\hline & De 30 a 59 & 38 & 74,6 & 54 & 55,7 & 18.394 & 56,4 \\
\hline & Com 60 anos ou mais & 4 & 7,9 & 18 & 18,6 & 7.186 & 22,0 \\
\hline \multirow[t]{4}{*}{ Nível de estudos } & Sem estudos & 0 & 0,0 & 6 & 6,2 & 17.330 & 44,9 \\
\hline & Ensino Fundamental & 14 & 27,5 & 15 & 15,5 & 7.156 & 18,5 \\
\hline & Ensino Médio & 20 & 39,2 & 43 & 44,3 & 9.189 & 23,8 \\
\hline & Ensino Superior & 17 & 33,3 & 33 & 34,0 & 4.934 & 12,8 \\
\hline \multirow[t]{3}{*}{ Classe social } & $A / B^{1}$ & 20 & 39,2 & 55 & 56,7 & 1.954 & 4,7 \\
\hline & $C / D / E^{2}$ & 31 & 60,8 & 42 & 43,3 & 40.035 & 95,3 \\
\hline & Total & 51 & 100,0 & 97 & 100,0 & 41.989 & 100,0 \\
\hline
\end{tabular}

Nota: ${ }^{1} \mathrm{~A} / \mathrm{B}$ : renda familiar acima de cinco salários-mínimos; ${ }^{2} \mathrm{C} / \mathrm{D} / \mathrm{E}$ : renda familiar de até cinco salários-mínimos. Fonte: dados da pesquisa. ${ }^{3}$ Instituto Brasileiro de Geografia e Estatística (2020).

É necessário enfatizar ao leitor que discrepâncias no perfil sociodemográfico dos consumidores, observadas entre as duas amostras, podem estar representadas nos dois grupos de consumidores que, na média, apresentam certa divergência nas preferências. Essa disparidade pode representar uma limitação do estudo, uma vez que, ao mensurar a relevância dos atributos (e a determinância de seus respectivos níveis) mais salientes, assumiu-se que a saliência dos atributos da carne bovina é praticamente igual entre os consumidores das duas amostras. Os resultados descritos nas próximas seções mostram uma forte inclinação por três

1 O valor do salário-mínimo em vigor a partir de 1o de janeiro de 2017 era de R\$937,00/mês (Decreto no 8.948/2016) (Brasil, 2016). 
atributos da carne bovina, o que sugere que as preferências médias dos consumidores das duas amostras não sejam tão expressivas a ponto de inviabilizar a pesquisa por suas suposições.

\subsection{SALIÊNCIA DOS ATRIBUTOS DA CARNE BOVINA}

Esta seção destina-se à apresentação dos resultados da análise da saliência dos atributos da carne bovina, avaliados para dois períodos: no momento da compra da carne bovina e na hora do consumo.

\subsubsection{Na compra}

A Tabela 4 apresenta a saliência dos atributos da carne bovina na compra do produto. Entre os 51 consumidores entrevistados, sete mencionaram haver três atributos salientes na compra da carne bovina, 25 apontaram dois atributos e os outros 19 consumidores citaram apenas um atributo. O número reduzido de menções de atributos era esperado, uma vez que as pessoas são incapazes ou estão indispostas a relacionar espontaneamente um conjunto extenso de atributos que possam estar influenciando sua percepção de qualidade dos produtos (Reynolds \& Olson, 2001).

No total, os entrevistados enumeraram 17 atributos como salientes. Como explicado anteriormente, o atributo citado primeiro pelo entrevistado foi tomado como o mais saliente pelo consumidor, logo o mencionado na sequência foi o segundo mais saliente e assim por diante.

Tabela 4. Saliência dos atributos da carne bovina no momento da compra

\begin{tabular}{clcccc} 
Ordem & \multicolumn{1}{c}{ Atributos } & $\mathbf{1}^{\circ}$ na ordem & $\mathbf{2}^{\circ}$ na ordem & $\mathbf{3}^{\circ} \mathbf{n a}$ ordem & Total \\
\hline 1 & Cor & 26 & 11 & 1 & 38 \\
2 & Aparência & 8 & 5 & 1 & 14 \\
3 & Gordura & 4 & 6 & 0 & 10 \\
4 & Higiene & 3 & 0 & 0 & 3 \\
4 & Preço & 3 & 0 & 0 & 3 \\
5 & Produto fresco & 2 & 2 & 0 & 4 \\
6 & Qualidade & 1 & 2 & 0 & 3 \\
7 & Tempo de prateleira & 1 & 1 & 2 & 4 \\
8 & Saudável & 1 & 0 & 0 & 1 \\
8 & Aspecto & 1 & 0 & 0 & 1 \\
8 & Procedência & 1 & 0 & 0 & 1 \\
9 & Atendimento & 0 & 1 & 1 & 2 \\
10 & Embalagem & 0 & 1 & 0 & 1 \\
10 & Textura & 0 & 1 & 0 & 1 \\
10 & Maciez & 0 & 1 & 0 & 1 \\
10 & Corte & 0 & 1 & 0 & 1 \\
11 & Cheiro & 0 & 0 & 2 & 2 \\
\end{tabular}

Fonte: dados da pesquisa.

No geral, a cor da carne bovina é o atributo mais saliente na hora da compra. Tal atributo foi indicado por 26 pessoas como o mais saliente, 11 pessoas o mencionaram como o segundo atributo mais saliente e somente uma pessoa o apontou como o terceiro mais saliente. Essas respostas convergem com os resultados de pesquisas prévias, como a de Gracia \& de Magistris (2013), Ngapo et al. (2007), Verbeke et al. (2005), em que descrevem que o consumidor "compra a carne com os olhos". Em Dracena, Groot (2020) verificou, por meio do EEDMP do caso 1, com 12 
atributos, que a aparência/cor é o atributo que os consumidores mais consideram no momento das compras. Pelo método de EL, a aparência é o segundo atributo mais saliente, pois obteve 14 citações, sendo oito menções como o mais saliente e cinco como o segundo mais saliente.

Assim como a cor, a quantidade de gordura é uma das dimensões da aparência da carne (Font-I-Furnols \& Guerrero, 2014). A gordura é o terceiro atributo mais saliente no momento da compra por ter sido indicado quatro vezes como o mais saliente e seis vezes como o segundo mais saliente. Além de estar relacionada com o aspecto da carne, a gordura intramuscular está associada a certos fatores post mortem que impactam a textura e a suculência da carne. Por essa razão, alguns consumidores desejam carne marmorizada (Juárez et al., 2012). Por outro lado, a gordura da carne está relacionada com problemas de sobrepeso e altas taxas de colesterol no sangue. Assim também existem seguimentos de consumidores que desejam carne magra em vez de marmorizada.

A higiene e o preço apresentam o mesmo grau de saliência (quarto lugar na ordem) porque ambos receberam três indicações como os atributos mais salientes, no entanto não receberam indicações como segundo ou mesmo terceiro atributo mais saliente. Desta forma, mesmo que o frescor seja classificado como o quinto atributo mais saliente, é, na verdade, o sexto.

A textura e a maciez são dois atributos de experiência e, ao contrário dos atributos de busca, o consumidor não consegue avaliá-los no ato das compras. Portanto, dizer que tais atributos são salientes na compra da carne é algo questionável, porém essas respostas não interferem significativamente na qualidade dos resultados. São apenas duas indicações inconsistente num total de 90 indicações.

\subsubsection{No consumo}

Os atributos de experiência são provados no consumo da carne bovina. Somente após o consumo, as pessoas estão aptas a julgar a discrepância entre a expectativa de qualidade e a qualidade observada do alimento. A discrepância interfere diretamente na satisfação do consumidor. Caso comprove que a qualidade do produto é igual ou superior à expectativa inicial, o consumidor ficará satisfeito e é bem provável que volte a comprar a carne bovina (Brunsø et al., 2002).

Neste sentido, a Tabela 5 mostra os atributos mais salientes quando os consumidores de Dracena consomem carne bovina. É possível verificar que quatro consumidores relacionaram três atributos como salientes, 23 consumidores indicaram dois atributos e 24 apontaram apenas um atributo. Ao final, foram relacionados dez atributos da carne bovina como salientes na hora do consumo.

O sabor da carne bovina é o atributo mais saliente no consumo. Trinta e dois consumidores mencionaram-no como o mais saliente e nove pessoas disseram ser o segundo atributo mais saliente. A maciez é o segundo atributo mais saliente para os consumidores. Foi indicada como mais saliente por dez consumidores e como o segundo mais saliente por 13 consumidores.

Embora haja grande diferença em relação aos dois atributos anteriores, a (quantidade) de gordura na carne bovina é o terceiro atributo mais saliente, pois foi eleito três vezes como o mais saliente. Para os consumidores, a aparência, a procedência, o frescor e a praticidade de preparo da carne são atributos com a mesma saliência. Todos esses quatro atributos receberam apenas uma indicação como o mais saliente (primeiro na ordem das respostas), portanto ocupam a quinta posição na ordem de importância. 
Tabela 5. Grau de saliência dos atributos no momento do consumo da carne bovina

\begin{tabular}{clcccc} 
Ordem & \multicolumn{1}{c}{ Atributos } & $\mathbf{1}^{\mathbf{0}} \mathbf{n a}$ ordem & $\mathbf{2}^{\mathbf{0}} \mathbf{n a}$ ordem & $\mathbf{3}^{\mathbf{0}} \mathbf{n a}$ ordem & Total \\
\hline 1 & Sabor & 32 & 9 & 0 & 41 \\
2 & Maciez & 10 & 13 & 2 & 26 \\
3 & Gordura & 3 & 0 & 0 & 3 \\
4 & Textura & 1 & 2 & 1 & 4 \\
5 & Aparência & 1 & 0 & 0 & 1 \\
5 & Procedência & 1 & 0 & 0 & 1 \\
5 & Produto fresco & 1 & 0 & 0 & 1 \\
5 & Praticidade no preparo & 1 & 0 & 0 & 1 \\
6 & Cheiro & 0 & 2 & 1 & 3 \\
7 & Produtos químicos & 0 & 1 & 0 & 1 \\
& Total & 51 & 27 & 4 & 82 \\
\hline
\end{tabular}

Fonte: dados de pesquisa.

\subsection{RELEVÂNCIA E DETERMINÂNCIA DOS ATRIBUTOS DA CARNE BOVINA}

Os resultados das preferências dos entrevistados pela relevância dos atributos da carne bovina e a determinância de seus respectivos níveis, obtidos pelo EEDMP do caso 2 (coluna EEDMP) e pelo experimento de intenção de compra da carne bovina (coluna Binário), estão expostos na Tabela 6. Em ambos os casos, foram estimadas regressões logísticas. A regressão do primeiro experimento foi calculada com base em 1.746 observações (97 entrevistados x 9 perfis de alcatra por entrevistado $\times 2$ escolhas por perfil), enquanto a regressão do segundo experimento foi estimada por 873 observações (97 entrevistados x 9 perfis de alcatra por entrevistado x 1 escolha por perfil).

O valor do Critério de Informação de Akaike (AIC) do EEDMP foi calculado em 4.487,7 e o McFadden Pseudo R², em 7,26\%, à medida que o valor do AIC do experimento binário de intenção de compras foi estimado em 1.087,2 e o McFadden pseudo R², em 11,03\%, o que sugere que o modelo binário pode ter um ajuste um pouco melhor aos dados que o EEDMP.

$\mathrm{O}$ valor do McFadden pseudo $\mathrm{R}^{2}$ está relacionado com a capacidade preditiva do modelo. Embora os valores McFadden pseudo $\mathrm{R}^{2}$ tendam a ser menores que os valores de $\mathrm{R}^{2}$ calculados por mínimos quadrados ordinários, um modelo moderadamente forte apresenta o McFadden pseudo $\mathrm{R}^{2}$ oscilando entre $15 \%$ e $20 \%$ (Hu et al., 2006). Desta forma, verifica-se que ambos os modelos apresentam baixa capacidade preditiva das escolhas dos consumidores. Isso se deve ao tamanho da amostra, ao comprometimento dos entrevistados com a qualidade de suas respostas e até mesmo à própria dificuldade de compreensão dos experimentos de escolha pelos entrevistados.

Tabela 6. Relevância dos atributos da carne bovina e determinância de seus respectivos níveis

\begin{tabular}{|c|c|c|c|}
\hline & Atributo e nível de atributo & EEDMP & Binário \\
\hline \multirow{4}{*}{ 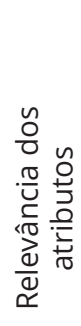 } & Constante & - & $\begin{array}{l}0,152^{\text {ns }} \\
(0,143)\end{array}$ \\
\hline & Preço & $\begin{array}{c}-1,592 * * * \\
(0,190)\end{array}$ & - \\
\hline & Cor & $\begin{array}{c}-0,143^{* *} \\
(0,070)\end{array}$ & - \\
\hline & Sabor & $\begin{array}{c}0,801 * * * \\
(0,070)\end{array}$ & - \\
\hline
\end{tabular}

ns: significa que o parâmetro estimado não apresentou diferença estatística significativa; * apresentou diferença estatística significativa a 10\%; ** apresentou diferença estatística significativa a 5\%; *** apresentou diferença estatística significativa a 1\%. Os números apresentados entre parênteses referem-se aos valores de erro-padrão dos respectivos coeficientes. Fonte: dados da pesquisa. 
Tabela 6. Continuação...

\begin{tabular}{|c|c|c|c|}
\hline & Atributo e nível de atributo & EEDMP & Binário \\
\hline \multirow{8}{*}{  } & Preço & $\begin{array}{c}0,055^{\star * *} \\
(0,007)\end{array}$ & $\begin{array}{c}-0,012 * \star \\
(0,006)\end{array}$ \\
\hline & Vermelho-brilhante & $\begin{array}{l}0,088^{\text {ns }} \\
(0,081)\end{array}$ & $\begin{array}{c}0,275^{* * *} \\
(0,063)\end{array}$ \\
\hline & & $0,152^{*}$ & $-0,115^{*}$ \\
\hline & vermeino-purpura & $(0,081)$ & $(0,067)$ \\
\hline & Bom sabor garantido & $\begin{array}{l}0,040^{\text {ns }} \\
(0,082)\end{array}$ & $\begin{array}{c}0,342 * * * \\
(0,063)\end{array}$ \\
\hline & Provavelmente não tem bom sabor & $\begin{array}{c}0,376 * * * \\
(0,083)\end{array}$ & $\begin{array}{c}-0,644 * * * \\
(0,068)\end{array}$ \\
\hline & Maciez garantida & $\begin{array}{l}0,139 * \\
(0,081)\end{array}$ & $\begin{array}{c}0,131 \text { ** } \\
(0,064)\end{array}$ \\
\hline & Provavelmente dura & $\begin{array}{l}0,018^{\text {ns }} \\
(0,081)\end{array}$ & $\begin{array}{c}-0,307 * * * \\
(0,067)\end{array}$ \\
\hline \multirow{5}{*}{ 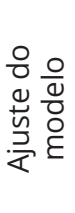 } & Função de máxima verossimilhança & $-2.413,48$ & -536 \\
\hline & McFadden pseudo $\mathrm{R}^{2}$ & $7,26 \%$ & $11,03 \%$ \\
\hline & Número de observações & 1.746 & 873 \\
\hline & Número de parâmetros estimados & 10 & 8 \\
\hline & AIC & $4.487,7$ & $1.087,2$ \\
\hline
\end{tabular}

ns: significa que o parâmetro estimado não apresentou diferença estatística significativa; * apresentou diferença estatística significativa a 10\%; ** apresentou diferença estatística significativa a 5\%; *** apresentou diferença estatística significativa a 1\%. Os números apresentados entre parênteses referem-se aos valores de erro-padrão dos respectivos coeficientes. Fonte: dados da pesquisa.

Embora as regressões tenham sido realizadas com diferentes bases de dados, representam preferências do mesmo grupo de pessoas. Por isso, a determinância dos dois modelos deveria representar uma estrutura de preferência. No entanto, os resultados da Tabela 6 mostram que isso não ocorre. As duas regressões indicam que os preços são determinantes nas decisões de compra da carne bovina (parâmetros estatisticamente diferentes de zero), no entanto a utilidade marginal dos preços é positiva no EEDMP $(0,055)$ e negativa nas intenções de compras $(-0,012)$. A utilidade marginal negativa está de acordo com a teoria econômica, pois, na condição ceteris paribus, quanto maior o preço do produto, menor a utilidade ao consumidor (Vasconcellos \& Garcia, 2014).

Os modelos estimados indicam diferentes preferências quanto ao sabor da carne. As intenções de compra mostram que a utilidade da carne bovina que provavelmente tem bom sabor é maior que a utilidade da carne que provavelmente não tem bom sabor e que a utilidade da carne com bom sabor garantido é superior à utilidade da carne que provavelmente tem bom sabor. Neste sentido, os consumidores não querem correr risco na compra da carne e preferem mais a carne saborosa. Esse resultado era o esperado, uma vez que estudos prévios, como os de Uys e Bisschoff (2016) e Li et al. (2015), relatam que os consumidores preferem carne bovina com bom sabor.

Os parâmetros estimados com base em EEDMP mostram que os consumidores de Dracena preferem a carne que provavelmente não tem bom sabor à carne que provavelmente tem bom sabor, ou seja, gostam mais da carne com sabor desagradável, o que está fora das expectativas iniciais da pesquisa. O parâmetro da utilidade marginal de bom sabor garantido $(0,040)$ é estatisticamente igual a zero, o que indica que os consumidores são indiferentes entre a carne certificada e a não certificada. Esse resultado poderia ser esperado, uma vez que o mercado de carne bovina certificada na cidade é pouco expressivo ou praticamente inexistente. 
Os dois modelos também mostram certas discrepâncias nas preferências por níveis de textura. O modelo estimado pelas intenções de compra (binário) mostra que a utilidade marginal da carne provavelmente dura em relação à carne provavelmente macia é negativa $(-0,307)$ e a utilidade da carne com maciez garantida é superior à utilidade da carne provavelmente macia $(0,131)$. Por outro lado, as utilidades marginais calculadas pelo EEDMP sugerem que os consumidores são indiferentes entre a carne provavelmente macia e a provavelmente dura (utilidade marginal: 0,018, sem significância estatística), porém preferem carne com maciez garantida à que é provavelmente macia. Os resultados se somam aos observados por Hanagriff et al. (2009), que realizaram estudo de preferências com os consumidores dos Estados Unidos. Entre os 13 atributos da carne bovina que eles estudaram, os consumidores consideraram garantia de maciez o principal requisito de qualidade.

As determinâncias dos níveis de cor da carne bovina, estimadas pelas duas regressões, também mostram disparidades. Enquanto as intenções de compra dizem que os consumidores preferem a carne vermelho-brilhante à carne marrom e a marrom à vermelho-púrpura, o modelo estimado pelo EEDMP indica que os consumidores são indiferentes entre a carne marrom e a vermelho-brilhante e preferem a carne vermelho-púrpura às outras duas cores. Os resultados do modelo binário estão mais alinhados aos observados por Correa et al. (2020), em Redenção (PA). Nessa cidade, ao redor de $57,2 \%$ das pessoas preferem carne fresca vermelho-brilhante, $25,7 \%$ consideram a carne vermelho-púrpura sua cor predileta para carne bovina e o restante $(17,3 \%)$ quer mais carne marrom.

De acordo com os resultados do EEDMP, o sabor é o atributo mais relevante da carne bovina. O parâmetro da relevância do sabor foi calculado em 0,801 (e é estatisticamente diferente de zero). O segundo atributo mais relevante é a maciez. O preço é o atributo menos relevante entre os estudados, uma vez que o parâmetro estimado do preço $(-1,592)$ é o mais negativo entre os parâmetros estudados. Os resultados apurados podem ser considerados semelhantes aos de saliência (Tabelas 4 e 5) e aos observados por Hui et al. (1995), nos Estados Unidos. Esses autores verificaram que o sabor é o atributo mais importante nas decisões de compras de carne bovina e é seguido, em importância, pela aparência e pela maciez da carne. O preço da carne é um dos atributos menos relevantes nas decisões de compra dos consumidores desse país.

\section{CONCLUSÕES}

O Brasil apresenta vantagens competitivas na criação de bovinos. Embora o mercado de carne bovina seja expressivo no país, seus produtos ainda são majoritariamente comercializados como commodity. Para superar essa situação, a primeira medida é conhecer as preferências dos consumidores para, assim, diferenciar e agregar valor.

A importância dos atributos intrínsecos e extrínsecos da carne bovina apresenta três dimensões: saliência, relevância e determinância. Embora a maioria dos estudos apenas avalie uma das dimensões - que pode repercutir na validade dos resultados desses trabalhos -, tradicionalmente se recomenda que as três dimensões sejam avaliadas por três métodos distintos. O presente estudo visou avaliar as três dimensões por meio de dois métodos: EL, para avaliar a saliência dos atributos, e EEDMP, do caso 2, para mensurar, simultaneamente, a relevância dos atributos e a determinância de seus respectivos níveis. A intenção era simplificar a participação dos entrevistados e melhorar a qualidade das respostas. No entanto, verificou-se que o EEDMP do caso 2 gerou resultados diferentes das declarações de intenção de compras dos consumidores de Dracena, bem como de estudos prévios na área. 
O EEDMP do caso 2 é amplamente utilizado em pesquisas de preferências de pacientes com sucesso. Assim, baseando-se na literatura e nos resultados dessa pesquisa, recomenda-se que haja novos estudos, com outros produtos, diferentes desenhos experimentais (que estão ligados à complexidade do experimento), em outras localidades e com amostras maiores, para verificar a existência de novas discrepâncias. A complexidade do questionário e a dificuldade de algumas pessoas para entendê-lo perfeitamente podem ter sido os fatores que geraram resultados tão fora do esperado. Com o que se apresenta, ainda não é possível fazer nenhuma recomendação de uso do EEDMP do caso 2 para avaliar as preferências por alimentos.

O trabalho também mostrou que a saliência dos atributos da carne bovina depende do momento no processo de compra. No momento da compra, quando os consumidores apenas conseguem avaliar os atributos de busca, o atributo mais importante é a cor da carne, sendo essa característica seguida pela aparência do produto. A expressão “o consumidor compra com os olhos" ganha mais sentido para os consumidores de Dracena. No momento do consumo, em que o consumidor é capaz de avaliar os atributos de experiência, o atributo da carne bovina mais saliente é o sabor e o segundo mais saliente é a maciez do alimento.

Embora tenhamos que ter cautela com os resultados do EEDMP, o experimento indicou que o atributo mais relevante da compra da carne bovina é o sabor e, na sequência, a textura ou a maciez. Os consumidores dão mais importância aos atributos de experiência, que determinam a qualidade organoléptica da carne. A cor da carne, que normalmente influencia a expectativa no que diz respeito à qualidade organoléptica do alimento durante a compra, é o terceiro atributo mais relevante no processo de compra. O atributo menos relevante é o preço.

As declarações de intenção de compra de carne bovina mostraram que os consumidores de Dracena gostam mais da carne vermelho-brilhante que marrom ou vermelho-púrpura, provavelmente por perceberem mais frescor do produto. Além disso, os consumidores gostam mais da carne bovina macia e saborosa e com garantia quanto a esses parâmetros de qualidade.

Ambos os atributos de qualidade são definidos, em grande medida, dentro da porteira, ou seja, na propriedade rural. Para melhorar esse aspecto, é preciso haver incentivos, via preços, aos pecuaristas para que adotem raças de bovinos que, por exemplo, apresentem bom marmoreio (sabor e textura) e criem esses animais com alimentação e manejo adequados. 0 incentivo poderia ser oferecido pelos frigoríficos, que teriam seus produtos valorizados pelo mercado consumidor mediante certificações que garantissem maciez, sabor e suculência da carne bovina aos consumidores.

\section{AGRADECIMENTOS}

Agradecemos à Fundação de Amparo à Pesquisa do Estado de São Paulo (FAPESP), processo $n^{\circ}$ 2017/00748-6, que culminou nesse trabalho de pesquisa.

\section{REFERÊNCIAS}

Aguiar, G. A. M. (2018). Análise da estrutura competitiva e comportamento estratégico das indústrias frigoríficas participantes do programa carne Angus certificada em São Paulo (Dissertação de mestrado). Universidade Estadual Paulista, Jaboticabal.

Aizaki, H., \& Fogarty, J. (2019). An R package and tutorial for case 2 best-worst scaling. Journal of Choice Modelling, 32, 100171. http://dx.doi.org/10.1016/j.jocm.2019.100171

Andrighetto, C., Jorge, A. M., Nasser, M. D., Maestá, S. A., Rodrigues, E., \& Francisco, C. L. (2010). Características químicas e sensoriais da carne bovina. Pubvet, 4, 780-787. 
Associação Brasileira das Indústrias Exportadoras de Carne - ABIEC. (2020). Beef Report 2020. Recuperado em 22 de dezembro de 2020, de http://abiec.com.br/publicacoes/beef-report-2020/

Benedict, J., Steenkamp, M. E., \& Van Trijp, H. C. M. (1997). Attribute elicitation in marketing research. Marketing Letters, 8(2), 153-165.

Brasil. (2016). Decreto n ${ }^{\circ} 8.948$, de 29 de dezembro de 2016, que dispõe sobre o valor do salário-mínimo e a sua política de valorização de longo prazo. Diário Oficial [da] República Federativa do Brasil, Brasília. Recuperado em 22 de dezembro de 2020, de http://www. normaslegais.com.br/legislacao/Decreto-8948-2016.htm

Brunsø, K., Fjord, T. A., \& Grunert, K. G. (2002). Consumers' food choice and quality perception. Recuperado em 22 de dezembro de 2020, de https://pure.au.dk/portal/files/32302886/wp77.pdf

Carpenter, C. E., Cornforth, D. P., \& Whittier, D. (2001). Consumer preferences for beef color and packaging did not affect eating satisfaction. Meat Science, 57, 359-363. http://dx.doi. org/10.1016/S0309-1740(00)00111-X

Collins, A. M., \& Loftus, E. (1975). A spreading activation theory of semantic processing. Psychological Review, 82, 407-428. http://dx.doi.org/10.1037//0033-295X.82.6.407

Correa, L.A.T., Calil Júnior, N.M., \& Carvalhal, M.V. de L. (2020). Qualidade sensorial da carne bovina maturada e in natura resfriada no município de Rendenção - PA. Nutritime, 17(4), 8755-8760.

Espinoza, F. S., \& Hirano, A. S. (2003). As dimensões de avaliação dos atributos importantes na compra de condicionadores de ar: um estudo aplicado. Revista de Administração Contemporânea, 7(4), 97-117. http://dx.doi.org/10.1590/S1415-65552003000400006

Finn, A., \& Louviere, J. J. (1992). Determining the appropriate response to evidence of public concern: the case of food safety. Journal of Public Policy \& Marketing, 11(2), 12-25.

Flores, D. F. (2016). Programa Carne Angus Certificada: procedimentos da certificação (Trabalho de Conclusão de Curso). Faculdade de Veterinária, Universidade Federal do Rio Grande do Sul, Porto Alegre.

Flynn, T. N., Louviere, J. J., Peters, T. J., \& Coast, J. (2007). Best-Worst scaling: what it can do for health care research and how to do it. Journal of Health Economics, 26, 171-189. http:// dx.doi.org/10.1016/j.jhealeco.2006.04.002

Font-I-Furnols, M., \& Guerrero, L. (2014). Consumer preference, behavior and perception about meat and meat products: an overview. Meat Science, 98(3), 361-371. http://dx.doi. org/10.1016/j.meatsci.2014.06.025

Garcia, S. F. A., De Carvalho, D. T., Miranda, C. S., \& Bosco, B. R. M. (2018). Quais os sinais de carne macia e saborosa? A análise dos atributos de carne bovina que, no momento da compra, melhor sinalizam os benefícios mais desejados pelo consumidor. Revista Brasileira de Marketing, 17(4), 487-501. http://dx.doi.org/10.5585/remark.v17i4.3758

Gracia, A., \& de Magistris, T. (2013). Preferences for lamb meat: a choice experiment for Spanish consumers. Meat Science, 95(2), 396-402. http://dx.doi.org/10.1016/j.meatsci.2013.05.006

Grebitus, C. (2008). Food quality from the consumer's perspective: an empirical analysis of perceived pork quality. Cuvillier.

Groot, E. (2020). The most relevant attributes on purchase decision of beef: a best-worst score approach. /Gepec, 24(2), 174-194.

Grunert, K. G. (2005). Food Quality and safety: consumer perception and demand. European Review of Agriculture Economics, 32(3), 369-391. 
Grunert, K. G., Bredahl, L., \& Brunsø, K. (2004). Consumer perception of meat quality and implications for product development in the meat sector - a review. Meat Science, 66, 259-272.

Gujarati, D. N. (2000). Econometria básica (846 p.). São Paulo: MAKRON Books.

Hanagriff, R., Rhoades, R., \& Wilmeth, D. (2009). Consumer preferences in purchasing beef and the values they attribute to branded beef products. Recuperado em 22 de dezembro de 2020, de https://core.ac.uk/download/pdf/6995669.pdf?repositoryld=153

Hu, B., Shao, J., \& Palta, M. (2006). Pseudo-R2 in logistic regression model. Statistica Sinica, 16(3), 847-860.

Hui, J., Mc Lean-Meyinsse, P. E., \& Jones, D. (1995). An empirical investigation of importance rating of meat attributes by Louisiana and Texas consumers. Journal of Agricultural and Applied Economics, 27(2), 636-643. http://dx.doi.org/10.1017/S1074070800028650

Instituto Brasileiro de Geografia e Estatística - IBGE. (2020). IBGE Cidades: Dracena. Recuperado em 22 de dezembro de 2020, de https://www.ibge.gov.br/estatisticas-novoportal/sociais/ saude/9050-pesquisa-de-orcamentos-familiares.html?=\&t=resultados

Jaccard, J., Brinberg, D., \& Ackerman, L. J. (1986). Assessing attribute importance: a comparison of six methods. The Journal of Consumer Research, 12, 463-468. http://dx.doi.org/10.1086/208530

Juárez, M., Aldai, N., López-Campos, Ó., Dugan, M. E. R., Uttaro, B., \& Aalhus, J. L. (2012). Beef Texture and Juiciness. In Y. H. Hui. Handbook of meat and meat processing (2nd ed., 1000 p.). Boca Raton: CRC Press.

Krech, D., \& Crutchfield, R. S. (1948). Theory and problems of social psychology. McGraw-Hill Book Co.

Lancsar, E., Louviere, J., Donaldson, C., Currie, G., \& Burges, L. (2013). Best worst discrete choice experiments in health: Methods and an application. Social Science \& Medicine, 76, 74-82. http://dx.doi.org/10.1016/j.socscimed.2012.10.007

Lelis, C. T., Teixeira, K. M. D., \& Silva, N. M. (2012). A inserção feminina no mercado de trabalho e suas implicações para os hábitos alimentares da mulher e de sua família. Saúde em Debate, 36(95), 523-532. http://dx.doi.org/10.1590/S0103-11042012000400004

Levordashka, A. (2006). Effects of questionnaire design on respondent experience and data quality (Dissertação de mestrado). VU University of Amsterdam, Amsterdam.

Li, X., Jensen, K. L., Clark, C. D., \& Lambert, D. M. (2015). Consumer willingness-to-pay for nontaste attributes in beef products. Recuperado em 22 de dezembro de 2020, de https:// ageconsearch.umn.edu/record/196719/

Marley, A. A. J., \& Flynn, T. N. (2015). Best worst scaling: theory and practice. In J. D. Wright (Ed.), International Encyclopedia of the social and behavioral science (2nd ed.). Elsevier.

Marley, A. A. J., \& Louviere, J. J. (2005). Some probabilistic models of best, worst, and best-worst choices. Mathematical Psychology, 49, 464-480. http://dx.doi.org/10.1016/j.jmp.2005.05.003

Marley, A. A. J., Flynn, T. N., \& Louviere, J. J. (2008). Probabilistic models of set-dependent and attribute-level best-worst choice. Journal of Mathematical Psychology, 52, 281-296. http:// dx.doi.org/10.1016/j.jmp.2008.02.002

Marotti, J., Galhardo, A. P. M., Furuyama, R. J., Pigozzo, M. N., Campos, T. N., \& Laganá, D. C. (2008). Amostragem em pesquisa clínica: tamanho da amostra. Revista de Odontologia da Universidade Cidade de São Paulo, 20(2), 186-194.

Menezes Filho, N., Oliveira, A. P., Rocha, R. H., \& Komatsu, B. (2016). Impacto do ensino superior sobre o trabalho e a renda dos municípios brasileiros. Recuperado em 22 de dezembro de 2020, de https://www.insper.edu.br/wp-content/uploads/2018/09//mpacto-Ensino-SuperiorTrabalho-Renda-Municipios-Brasileiros.pdf 
Mühlbacher, A. C., Kaczynski, A., Zweifel, P., \& Reed Johnson, F. (2016). Experimental measurement of preferences in health and healthcare using best-worst scaling: an overview. Health Economics Review, 6(2), http://dx.doi.org/10.1186/s13561-015-0079-x

Myers, J. H., \& Alpert, M. I. (1977). Semantic Confusion in Attitude Research: Salience Vs. Importance Vs. Determinance. Advances in Consumer Research. Association for Consumer Research (U. S.), 4, 106-110.

Ngapo, T. M., Martin, J.-F., \& Dransfield, E. (2007). International preferences for pork appearance: II. Factors influencing consumer choice. Food Quality and Preference, 18(1), 139-151. http:// dx.doi.org/10.1016/j.food.2005.09.007

Passetti, R. A. C., Torrecilhas, J. A., Ornaghi, M. G., Mottin, C., \& Guerrero, A. (2016). Determinação da coloração e a disposição de compra pelos consumidores de carne bovina. Publicações em Medicina Veterinária e Zootecnia, 10(2), 179-189. http://dx.doi.org/10.22256/pubvet.v10n2.179-189

Reynolds, T. J., \& Olson, J. C. (2001). Understanding consumer decision making: The means-end approach to marketing and advertising strategy. Psychology Press.

Ries, J. E. (2010). Atributos valorizados pelos consumidores em relação à carne bovina e ao ponto de venda - estudo de caso da Aproccima (Trabalho de Conclusão de Curso). Universidade do Vale dos Rios Sinos, São Leopoldo.

Schnettler, B., Fica, D., Sepúlveda, N., Sepúlveda, J., \& Denegri, M. (2010). Valoración de atributos intrínsecos y extrínsecos en la compra de la carne bovina en el sur de Chile. Revista Científica (Maracaibo), 20(1), 101-109.

Tirado, G. (2009). Demandas tecnológicas da cadeia produtiva da carne bovina: uma análise no estado de São Paulo. (Dissertação de mestrado). Faculdade de Agronomia e Medicina Veterinária, Universidade de Brasília, Brasília.

Trespalacios, J. A., Vázques, R., \& Bello, L. (2005). Investigación de mercados. Thompson.

Uys, P., \& Bisschoff, C. (2016). Identifying consumer buying preferences of beef. Problems and Perspectives in Management, 14(4), 256-263. http://dx.doi.org/10.21511/ppm.14(4-1).2016.15

Uzunoz, M., \& Akcay, Y. (2012). A case study of Probit model analysis of factors affecting consumption of packed and unpacked milk in Turkey. Economics of Agricultural and Food Markets. 2012, 732583. http://dx.doi.org/10.1155/2012/732583

Van Ittersum, K., Pennings, J. M. E., Wansink, B., \& Van Trijp, H. C. M. (2007). The validity of attribute-importance measurement: a review. Journal of Business Research, 60(11), 11771190. http://dx.doi.org/10.1016/j.jbusres.2007.04.001

Van Kleef, E., Van Trijp, H. C. M., \& Luning, P. (2005). Consumer research in the early stages of new product development: a critical review of methods and techniques. Food Quality and Preference, 16(3), 181-201. http://dx.doi.org/10.1016/j.foodqual.2004.05.012

Vasconcellos, M. A. S., \& Garcia, M. E. (2014). Fundamentos de economía (5. ed.). São Paulo: Saraiva.

Verbeke, W., de Smet, S., Vackier, I., Van Oeckel, M. J., Warnants, N., \& Van Kenhove, P. (2005). Role of intrinsic search cues in the formation of consumer preferences and choice for pork chops. Meat Science, 69(2), 343-354. https://doi.org/10.1016/j.meatsci.2004.08.005 